\title{
Predictive Value of Lidocaine for Treatment Success of Oxcarbazepine in Patients with Neuropathic Pain Syndrome
}

\author{
Sivan Schipper • Andreas R. Gantenbein • \\ Konrad Maurer $\cdot$ Eli Alon $\cdot$ Peter S. Sándor
}

To view enhanced content go to www.paintherapy-open.com

Received: October 19, 2012 / Published online: February 15, 2013

(c) The Author(s) 2013. This article is published with open access at Springerlink.com

\section{ABSTRACT}

Introduction: Pharmacotherapy in patients with neuropathic pain syndromes (NPS) can be associated with long periods of trial and error before reaching satisfactory analgesia. The aim of this study was to investigate whether a short intravenous (i.v.) infusion of lidocaine may have a predictive value for the efficacy of oxcarbazepine.

\section{S. Schipper}

Department of Internal Medicine,

Maennedorf Hospital, Maennedorf, Switzerland

A. R. Gantenbein

Neurology Outpatient Clinic, RehaClinic Bad

Zurzach, Bad Zurzach, Switzerland

\section{A. R. Gantenbein}

Department of Neurology, Headache and Pain Unit, University Hospital Zurich, Zurich, Switzerland

K. Maurer

Institute of Anaesthesiology,

University Hospital of Zurich, Zurich, Switzerland
Methods: In total, 16 consecutive patients with NPS were studied in a prospective, uncontrolled, open-label study design. Each patient received i.v. lidocaine $(5 \mathrm{mg} / \mathrm{kg})$ within $30 \mathrm{~min}$ followed by a long-term oral oxcarbazepine treatment (900-1,500 mg/day). During an observation period of 28 days, treatment response was documented by a questionnaire including the average daily pain score documented on a numeric rating scale (NRS).

\section{K. Maurer}

Institute of Physiology and Zurich Centre for Integrative Human Physiology (ZIHP), University of Zurich, Zurich, Switzerland

E. Alon

Private Pain Practice, Zurich, Switzerland

P. S. Sándor $(\square)$

ANNR RehaClinic, Cantonal Hospital Baden, Im Ergel, 5404 Baden, Switzerland e-mail: Peter.S.Sandor@gmail.com 
Results: A total of 6 out of 16 patients (38\%) were lidocaine responders (defined as pain reduction $>50 \%$ during the infusion), and 4 of $16(25 \%)$ were oxcarbazepine responders. In total, 6 out of 16 participants (38\%) discontinued oxcarbazepine treatment due to side effects. In an interim analysis predictive value of the lidocaine infusion was low with a Kendall's tau correlation coefficient of 0.29 and coefficient of determination $\mathrm{R}^{2}$ of 0.119 (95\% confidence interval -0.29 to 0.72 ). As a consequence of this low correlation, the study was discontinued for ethical reasons.

Conclusion: In conclusion, lidocaine infusion has a low predictive value for effectiveness of oxcarbazepine-if at all.

Keywords: Lidocaine; Neuropathic pain; Oxcarbazepine; Predictive value; Treatment response

\section{INTRODUCTION}

Treatment of neuropathic pain syndrome (NPS) constitutes a big challenge for the patient, for the physician, and for the whole public health system [1]. A multitude of drugs are recommended in the treatment of NPS [2]. Although there are a number of recommendations for the treatment of NPS [3-5], no clear criteria exist as to what drug should be used to initiate therapy in a certain patient.

Sodium channel inhibitors such as lidocaine and oxcarbazepine are used in the treatment of neuropathic pain $[6,7]$. Studies that have looked at the role of systemic lidocaine for predicting subsequent response to mexiletine showed a weak predictive value $[8,9]$. In contrast to mexiletine, which is a class IB antiarrhythmic drug and not licenced in Switzerland, oxcarbazepine is a sodium channel blocker which does not have the potential for significant cardiac side effects. Carbamazepine and oxcarbazepine are mostly considered thirdline drugs for the treatment of NPS [6]. Compared to carbamazepine, oxcarbazepine has less side effects and is better tolerated [10]. Oxcarbazepine was therefore considered the best choice to be used in the setting of this study. The aim of this study was to investigate whether the response to lidocaine may predict the therapeutic efficacy of oxcarbazepine.

\section{METHODS}

\section{Subjects and Study Design}

A prospective and uncontrolled open-label study design was used. The study was approved by the local ethical authorities. All procedures followed were in accordance with the ethical standards of the responsible committee on human experimentation (institutional and national) and with the Helsinki Declaration of 1975, as revised in 2008. Informed consent was obtained from all patients for being included in the study. Based on the published data of Galer et al. [8], a power analysis was performed that planned to include 30 patients. Participants were recruited from outpatients at the Department of Neurology and the Pain Clinic of the Institute of Anaesthesiology, University Hospital Zurich. The diagnosis of NPS was made either by a certified neurologist and confirmed by a certified anesthetist, or the other way round. Each participant was diagnosed by clear clinical criteria, including suggestive history, pain presentation in a certain body area, and the coexistence of positive symptoms (namely paresthesias, dysesthesias, spontaneous pain, allodynia, and hyperalgesia), and negative symptoms (namely hypoesthesia, hypoalgesia, 
and thermhypesthesia). All patients had NPS of mainly peripheral origin; these patients are known to be more responsive to treatment than patients with NPS of mainly central origin. Each participant filled in a questionnaire, supplying information about the NPS. Inclusion criteria were clinical diagnosis of NPS, age $>18$ years, and an average intensity of pain score of at least 5 according to an 11-point numeric rating scale (NRS; $0=$ no pain; $10=$ maximum pain imaginable) [11]. Exclusion criteria were intellectually or mentally impaired subjects, medical contraindication to lidocaine or oxcarbazepine, pregnancy, and/or antineuropathic comedication.

According to a standard protocol, the lidocaine infusion was given over $30 \mathrm{~min}$ at a dosage of $5 \mathrm{mg} / \mathrm{kg}$ body weight [12]. Pain measure (NRS) and reported side effects were documented, and minimal pain score reached during infusion time was used for statistical outcome. Subjects were started on oxcarbazepine, initially administered with a fixed scheme (day 1 and 2: $60 \mathrm{mg} /$ day; day 3 and 4: $120 \mathrm{mg} /$ day; day 5 and 6: $240 \mathrm{mg} /$ day; day 7 and 8: $300 \mathrm{mg} /$ day; day 9 and 10: $450 \mathrm{mg} /$ day; day 11-14: $600 \mathrm{mg} /$ day). Thereafter, the titration was individual according to efficacy and tolerability; the average maintenance dosage was between 900 and $1,500 \mathrm{mg}$ oxcarbazepine per day. The observation period was 28 days, during which NRS and side effects were documented daily by the patients on a standardized form. For statistical outcome, the minimal daily NRS reached by the patient during the observation period was used.

\section{Clinical Measures and Statistical Analysis}

Treatment success (responders) was defined as a reduction of NRS of $50 \%$ or more and treatment failure (nonresponders) as a pain reduction of less than 50\% [13]. Treatment efficacy was measured by post/pre-ratio. The correlation was calculated using Kendall's rank test and Fisher's z-transformation for 95\% confidence interval (CI). Kendall's tau correlation coefficient and the coefficient of determination were used to express the predictive value of the lidocaine test, with the latter test in a version with few predictions and therefore allowing for negative values.

Noticing the high dropout rate of oxcarbazepine treatment due to adverse effects, ethical questions about the continuation of the study were raised amongst the authors. Therefore, an unplanned interim analysis of the data and a post hoc power analysis were performed to estimate the sample size needed to find conclusive answers in the patient population based on the data set. Using $\chi^{2}$ test of equal proportions based on the data from the 16 included patients and to get a power of $80 \%$ with a test significance level of 0.05 with a onesided test, a sample size of 51 would have been necessary.

\section{RESULTS}

According to the adjusted sample size calculation based on the study sample, which required 51 subjects to obtain adequate power to prove a negative result, the study was stopped due to ethical reasons. To this point, a total of 19 patients had been enrolled in the study. Three participants were excluded: one because of an asthma attack during lidocaine infusion, and two because of incomplete documentation forms during the observation period, resulting in 16 participants that could be analyzed. The male:female ratio was 12:4, median age was $51 \pm 16$ years, and median duration of symptoms was $4.0 \pm 2.8$ years. For characteristics of patients and pain profiles, see Table 1. 


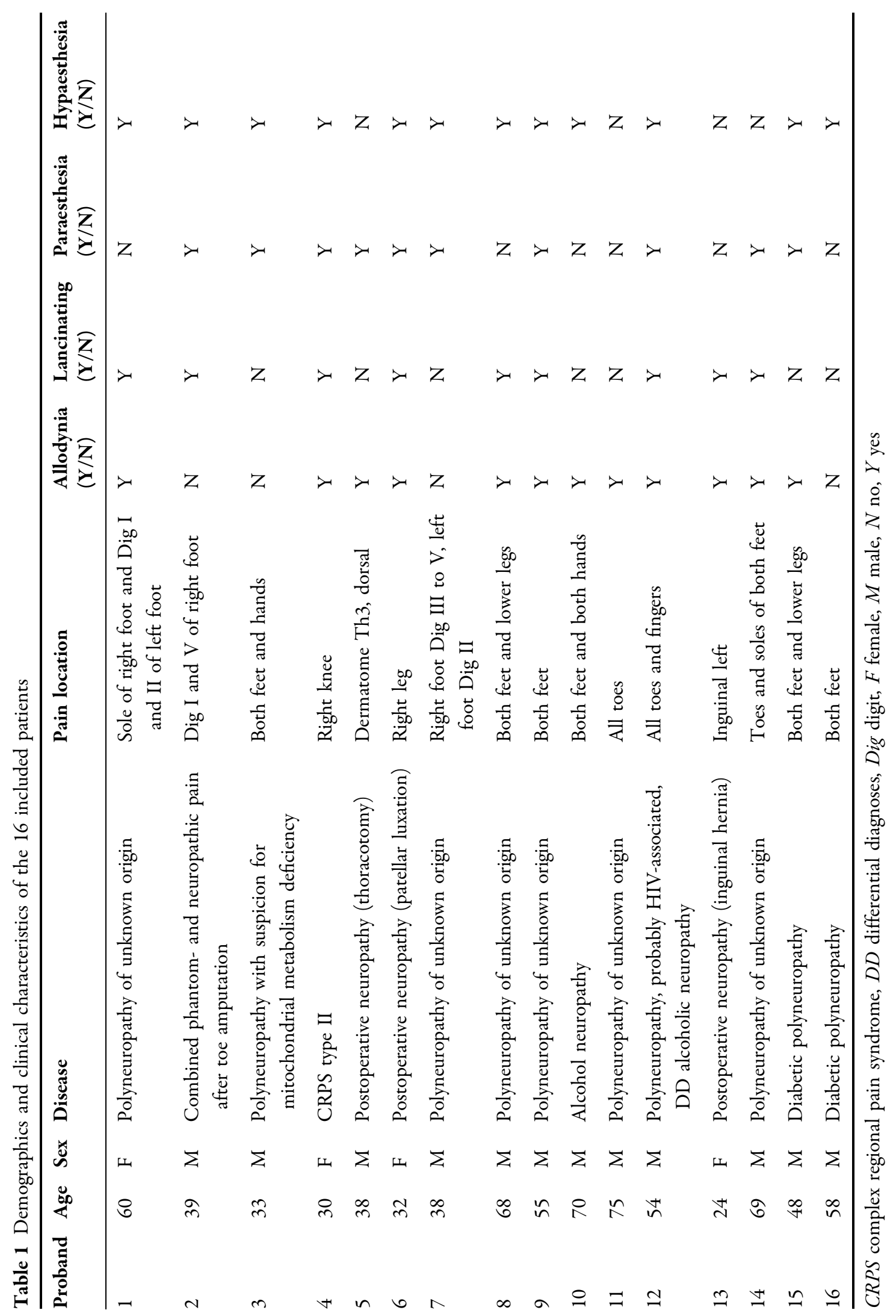




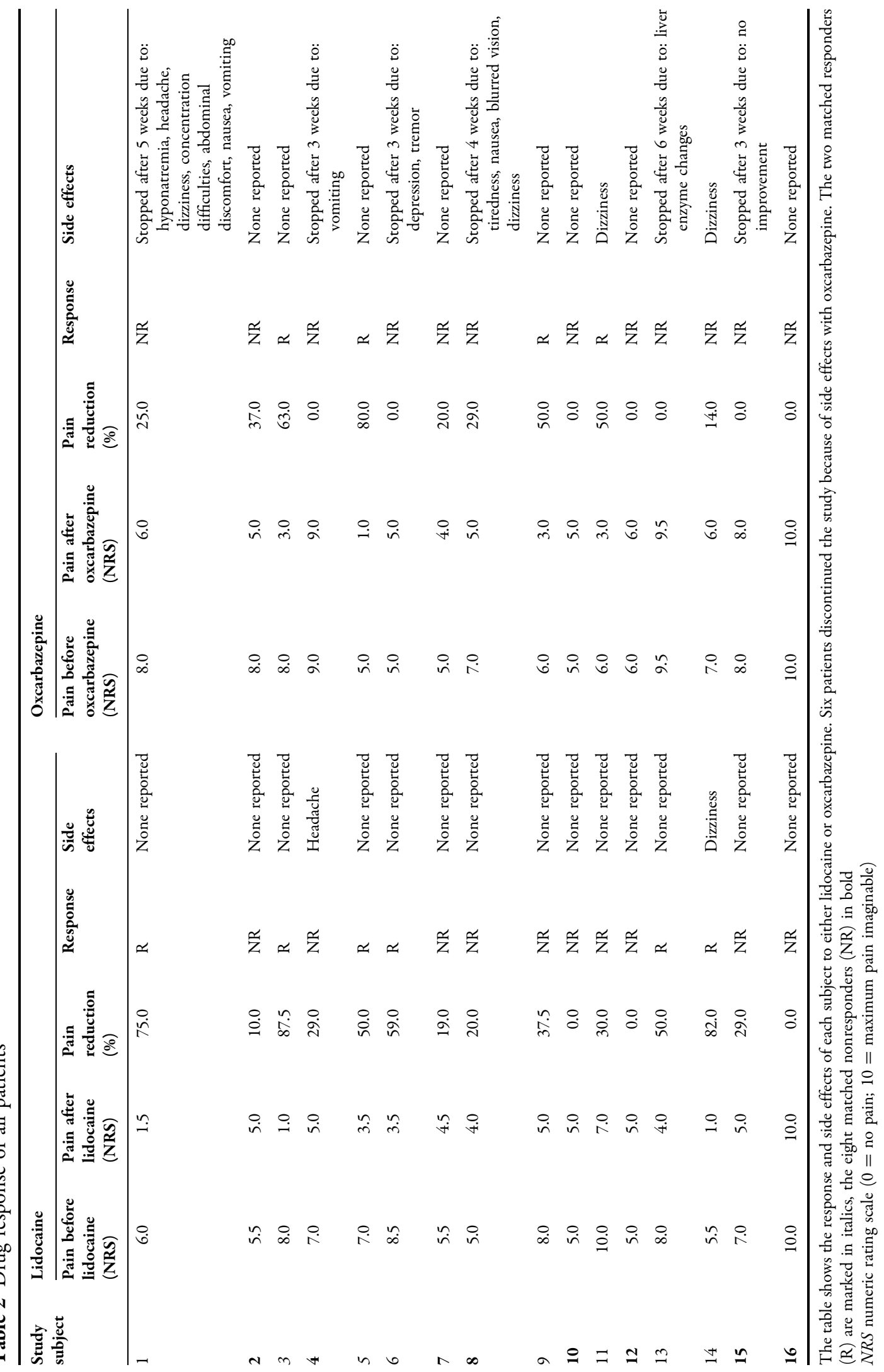




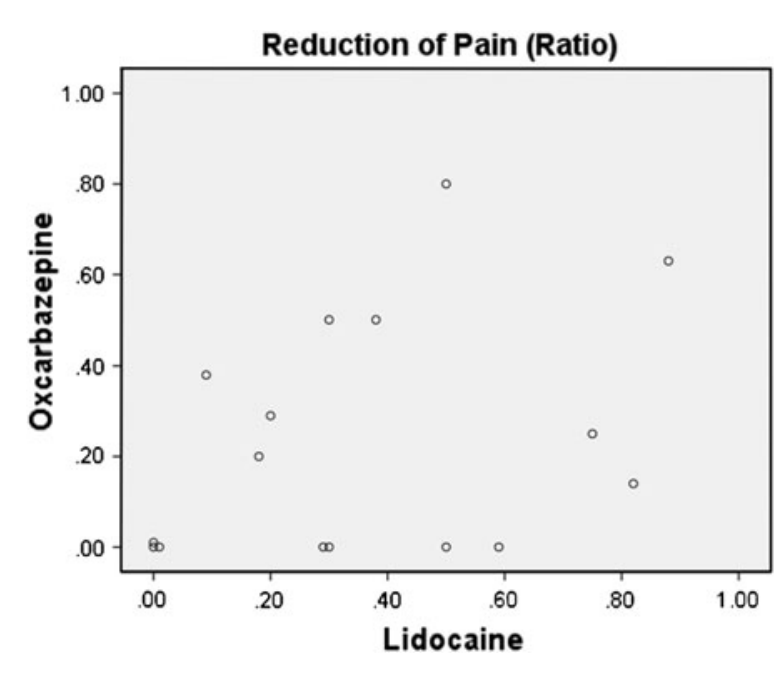

Fig. 1 Correlation of drug response. The figure shows that there was no correlation between the pain reduction to lidocaine and oxcarbazepine. Kendall's tau $=0.31$, $\mathrm{R}^{2}=0.119,95 \%$ confidence interval -0.29 to 0.72

In general, lidocaine infusion was well tolerated. During oxcarbazepine treatment, 8 out of 16 patients (50\%) reported side effects; 6 of them stopped oxcarbazepine treatment due to side effects.

Six patients (38\%) were lidocaine responders, and 4 out of the 16 subjects (25\%) responded to oxcarbazepine. Table 2 provides a summary of the results.

As shown in Fig. 1, there was no correlation between the change in pain while taking lidocaine and oxcarbazepine (Kendall's tau $=0.31, \mathrm{R}^{2}=0.119,95 \% \mathrm{CI}-0.29$ to 0.72 ).

\section{DISCUSSION}

The assumption that lidocaine infusion may reduce neuropathic pain but is ineffective in nonneuropathic pain was already made back in the 1980s [14]. Galer et al. [8] were the first to assume that if neuronal sodium channel inhibition was an important mechanism for relieving neuropathic pain, then different methods of producing this inhibition should produce similar degrees of pain relief. They tested the predictive value of lidocaine infusions for the effectivity of mexiletine in a small study of nine patients with polyneuropathy of various etiologies [8]. They postulated a significant correlation between the efficacies of both drugs, proposing to use intravenous lidocaine as a predictive test for the efficacy of analogous oral substances. However, the correlation was rather low in this study (Kendall's tau $=0.58$ ) as well as a subsequent study by Attal et al. [9], in which the correlation of lidocaine and mexiletine in their ability to reduce static mechanical allodynia was evaluated (Kendall's tau $=0.62$ ).

This study aimed to investigate the predictive value of lidocaine infusion for the efficacy of oxcarbazepine choosing a substance which is widely used in Switzerland, both for the treatment of epilepsy and NPS. Investigating 16 consecutive patients, the responder rate to lidocaine infusions was 38\% and the responder rate to oxcarbazepine was $25 \%$. These results are, although low, within the range of efficacy reported in previous studies $[12,15]$. However, $50 \%$ of patients experienced side effects due to oxcarbazepine, and the dropout rate was $38 \%$. Therefore, we performed an interim analysis in which Kendall's tau correlation coefficient was low and by far not significant. These interim data were far less promising than the previously published data and raised serious concerns about (1) the benefit of the oral oxcarbazepine treatment in our patient population, and (2) the predictive value of lidocaine. An adjusted interim sample size calculation was computed, which revealed a total number of 51 subjects to reach the defined significance levels (one-sided statement) and therefore prove, with a false negative error (=power) of 0.8 , that lidocaine has no predictive value for the response to 
oxcarbazepine. Lacking a minimum number of 35 participants, and since the lidocaine test is an invasive procedure with potentially dangerous side-effects such as cardiac arrhythmias, with the benefit of oxcarbazepine in our patient group being very limited and hampered by significant side effects, the decision was made to discontinue the study for ethical reasons.

To balance patient interests against the need for acquiring evidence is sometimes difficult for researchers. Nonetheless, it is ethically correct and considered "state of the art" in clinical research to stop a study as soon as convincing evidence that a new tool is not beneficial becomes available [16].

As in all studies with a comparable design, we cannot entirely exclude that the present results may have been biased by a placebo effect of lidocaine (and oxcarbazepine) or a nocebo effect or an interaction between the two sessions. In contrast to Attal et al. [9] we had decided not to blind the lidocaine infusion, as we found that patients in the clinical setting had reported slight paresthesias and dizziness, which they described spontaneously, making blinding, in our view, far less valuable.

In conclusion, we could not confirm our hypothesis, that lidocaine infusion can be used as a predictive test for effectiveness of oxcarbazepine, and prematurely aborted the study for ethical reasons following an interim analysis and a post hoc power calculation which revealed a far larger sample size compared to the first power calculation.

\section{ACKNOWLEDGMENTS}

Dr. Sándor is the guarantor for this article, and takes responsibility for the integrity of the work as a whole.
Conflict of interest. Dr. Sándor declares he has no conflict of interest. Dr. Schipper declares he has no conflict of interest. Dr. Gantenbein declares he has no conflict of interest. Dr. Maurer declares he has no conflict of interest. Prof. Alon declares he has no conflict of interest.

Open Access. This article is distributed under the terms of the Creative Commons Attribution Noncommercial License which permits any noncommercial use, distribution, and reproduction in any medium, provided the original author(s) and the source are credited.

\section{REFERENCES}

1. Berger A, Dukes EM, Oster G. Clinical characteristics and economic costs of patients with painful neuropathic disorders. J Pain. 2004;5:143-9.

2. Jensen TS, Madsen CS, Finnerup NB. Pharmacology and treatment of neuropathic pains. Curr Opin Neurol. 2009;22:467-74.

3. Dworkin $\mathrm{RH}, \mathrm{O}^{\prime}$ Connor $\mathrm{AB}$, Audette $\mathrm{J}$, et al. Recommendations for the pharmacological management of neuropathic pain: an overview and literature update. Mayo Clin Proc. 2010; 85(Suppl. 3):3-14.

4. Attal N, Cruccu G, Baron R, et al. EFNS guidelines on the pharmacological treatment of neuropathic pain: 2010 revision. Eur J Neurol. 2010;17:1113-88.

5. Finnerup NB, Otto M, McQuay HJ, Jensen TS, Sindrup SH. Algorithm for neuropathic pain treatment: an evidence based proposal. Pain. 2005;118:289-305.

6. Dworkin $\mathrm{RH}, \mathrm{O}^{\prime}$ Connor $\mathrm{AB}$, Backonja $\mathrm{M}$, et al. Pharmacologic management of neuropathic pain: evidence-based recommendations. Pain. 2007;132: 237-51.

7. Magenta P, Arghetti S, Di Palma F, et al. Oxcarbazepine is effective and safe in the treatment of neuropathic pain: pooled analysis of seven clinical studies. Neurol Sci. 2005;26:218-26.

8. Galer BS, Harle J, Rowbotham MC. Response to intravenous lidocaine infusion predicts subsequent response to oral mexiletine: a prospective study. J Pain Symptom Manage. 1996;12:161-7. 
9. Attal N, Rouaud J, Brasseur L, Chauvin M, Bouhassira D. Systemic lidocaine in pain due to peripheral nerve injury and predictors of response. Neurology. 2004;62:218-25.

10. Dam M, Ekberg R, Løyning Y, Waltimo O, Jakobsen K. A double-blind study comparing oxcarbazepine and carbamazepine in patients with newly diagnosed, previously untreated epilepsy. Epilepsy Res. 1989;3:70-6.

11. Breivik H, Borchgrevink PC, Allen SM, et al. Assessment of pain. Br J Anaesth. 2008;101:17-24.

12. Tremont-Lukats IW, Challapalli V, McNicol ED, Lau J, Carr DB. Systemic administration of local anesthetics to relieve neuropathic pain: a systematic review and meta-analysis. Anesth Analg. 2005; 101:1738-49.
13. Cepeda MS, Africano JM, Polo R, Alcala R, Carr DB. What decline in pain intensity is meaningful to patients with acute pain? Pain. 2003;105:151-7.

14. Boas RA, Covino BG, Shahnarian A. Analgesic responses to i.v. lignocaine. $\mathrm{Br} \mathrm{J}$ Anaesth. 1982;54:501-5.

15. Sindrup SH, Jensen TS. Efficacy of pharmacological treatments of neuropathic pain: an update and effect related to mechanism of drug action. Pain. 1999;83:389-400.

16. Freidlin B, Korn EL, Gray R. A general inefficacy interim monitoring rule for randomized clinical trials. Clin Trials. 2010;7:197-208. 